\title{
A molecular dynamics calculation of solid phase of malonic acid: role of hydrogen-bond chains and the elastic constants ${ }^{\dagger}$
}

\author{
SATHYA S R R PERUMAL ${ }^{\mathrm{a}, \mathrm{b}}$ and YASHONATH SUBRAMANIAN ${ }^{\mathrm{a}, \mathrm{b}, *}$ \\ ${ }^{a}$ Solid State and Structural Chemistry Unit, Indian Institute of Science, Bangalore, Karnataka 560 012, India \\ ${ }^{b}$ Thematic Unit of Excellence - Centre for Computational Material Science, Indian Institute of Science, \\ Bangalore, Karnataka 560 012, India \\ E-mail: yashonath@gmail.com
}

MS received 31 January 2017; revised 30 April 2017; accepted 1 May 2017

\begin{abstract}
Recent studies suggest that hydrogen bonds, in particular, hydrogen bond chains play an important role in determining the properties of a substance. We report an investigation into the triclinic phase of crystalline malonic acid. One of two intermolecular interaction potentials proposed here is seen to predict the lattice parameters as well as the enthalpy of the triclinic phase in good agreement with experimental data. Structural and dynamic properties are reported. Also reported are the lifetime of the hydrogen bond and hydrogen bond chains of length $l$ along [011] direction where $l=1$ to 5. From the temperature dependence of the lifetime we have obtained the activation energies of the chains. We also report the elements of elastic constant tensor. The results show that the presence of the hydrogen bond chain along [011] direction leads to higher value for elastic tensor $\mathrm{C}_{y y z z}$ suggesting a strong correlation between hydrogen bond chains and the elastic constant along that direction. This is consistent with the recent report of Azuri I et al. 2015 Angew. Chem. Int. Ed. Engl. 5413566 who reported that rather large Young's modulus for certain amino acid crystals.
\end{abstract}

Keywords. Hydrogen bond chain; elastic constants; molecular dynamics.

\section{Introduction}

There has been an increase in interest in trying to understand the role hydrogen bond plays in various properties of matter. In recent times, there have been several reports where hydrogen bond has played a key role. For example, Hu et al., control the shapeshifting of shape memory alloys by strain-induced, time-dependent reorganization of reversible hydrogen bonds dual network hydrogels. ${ }^{2}$ Monk et al., have found a relationship between the thermal and mechanical properties in phenolic resins. ${ }^{3}$ They found intra-chain as well as inter-chain hydrogen bonds formed a percolating 3D network and this is responsible for different elastic properties of the system. Davies et al., studied octacalcium phosphate where they found that the hydrogen bonds between the hydrated water as well as between water and calcium phosphate gave it higher mechanical strength to the structure. ${ }^{4}$ This has been confirmed by another study investigating the strength of the cement and calcium silicate hydrates. ${ }^{5}$

\footnotetext{
*For correspondence

${ }^{\dagger}$ Dedicated to the memory of the late Professor Charusita Chakravarty.
}

All these studies indicate the multifaceted role of hydrogen bonds in a wide variety of materials. Although these studies show a role for hydrogen bonds, a more unambiguous relationship between hydrogen bonds and other properties, in particular, mechanical property of the material is not evident.

Hydrogen bonds between molecules in the crystalline or liquid phase can extend over large distances and they can be three dimensional (as in water) or two dimensional (as in water confined between layeres) or one dimensional (as in water confined to a nanotube). Understanding of such networks and their properties help us to obtain insight into the properties of those materials themselves. It is well-known that properties of water such as heat of sublimation, anomalous thermal expansion, etc are predominantly influenced by these network of hydrogen bonds. Percolation in such network of hydrogen bonds is important and has been investigated in water using molecular dynamics simulations. ${ }^{6,7}$ Proton conduction in solids and liquids depends on the existence of such network of hydrogen bonds. Recently, proton conductivity in a co-crystal of gallic acid and isonizid was recently investigated through experimental measurements and density functional theory. ${ }^{8}$ Before 
the availability of Newtonian trajectories based simulations, formation or existence of percolation paths have also been analyzed by geometrical and mathematical models. Stanley and Teixeira have used percolation theory - a probabilistic model to determine the hydrogen bonds within the lattice - to propose liquid water model. ${ }^{9}$ Graph and set theory based approaches have been used to model an ordered network of hydrogen bonds. ${ }^{10,11}$ Hydrogen bond networks play an important role in determining many structural properties. Hydrogen bond mediated graphene oxide has been shown to determine mechanical properties of the functional materials. ${ }^{12,13}$ A recent study by Azuri et al., ${ }^{1}$ investigated the Young's moduli of amino acid crystals and found that the hydrogen bond networks can lead to large values, as large as 70-90 GPa for Young's moduli.

In order to understand and explore the relation between hydrogen bonds and mechanical properties, the choice of a simpler system would be appropriate. Dithiols, diamines, diols as well as dicarboxylic acids with functional groups at the two ends and methylene groups in between form an interesting series in which the physical properties alternate. ${ }^{14-23}$ Two molecules of diacids can form either a single or a double bond and therefore the strength of hydrogen bonds can vary.

We first propose two potentials for simulation of malonic acid. The properties computed show one of them to be able to predict experimental lattice parameters and enthalpy as well as structure. Subsequently, we study in detail the properties of hydrogen bond and chains of varying lengths. We report lifetimes as well as activation energies for chains. Elements of elastic constant tensor are then reported. These are then interpreted in terms of the hydrogen bond chains along certain crystallographic directions.

\section{Methods}

Molecular dynamics simulations were carried out in the isothermal-isobaric ensemble (NPT) with variable shape simulation cell of Parrinello and Rahman. ${ }^{24}$ In this, the simulation cell is defined by three vectors $\bar{a}, \bar{b}, \bar{c}$. These are included in the integration and their time evolution permits the simulation cell to change to any of the seven crystal systems.

Briefly, the Lagrangian is given by

$$
\begin{aligned}
L= & \frac{1}{2} \sum m_{i} \dot{\alpha}_{i}^{\prime} \underline{h^{\prime} h} \dot{\alpha}_{i}-\sum_{i} \sum_{j>i} \phi\left(r_{i j}\right) \\
& +\frac{1}{2} W \operatorname{Tr}\left(\underline{h}^{\prime} \underline{\hat{h}}\right)-p_{\text {ext }} V
\end{aligned}
$$

$\underline{h}$ is the matrix formed by column vectors $\mathbf{a}, \mathbf{b}, \mathbf{c}, \alpha_{i}$ is position of the ith particle in scaled coordinates and is given by $\mathbf{r}_{i}=\underline{h} \alpha_{i}$ Further, $r_{i j}^{2}=\left(\alpha_{i}-\alpha_{j}\right)^{\prime} \underline{G}\left(\alpha_{\mathbf{i}}-\alpha_{\mathbf{j}}\right)$ and $p_{\text {ext }}$ is the externally applied hydrostatic pressure, $\phi(r)$ is the pair potential, $W$ has the dimensions of mass and $V$ is volume of the MD cell $V=\operatorname{det}\{\underline{h}\}$

The equations of motions are

$$
\ddot{\alpha}_{i}=\frac{1}{m_{i}} \sum_{j \neq i} \chi\left(r_{i j}\right)\left(\alpha_{i}-\alpha_{j}\right)-\underline{G}^{-1} \underline{\dot{G}} \dot{\alpha}_{i},
$$

$\chi$ denotes $-d \phi / r d r$; and $\ddot{h}=W^{-1}\left(\pi-p_{\text {ext }}\right) \underline{\sigma}$ where matrix $\underline{\sigma}$ has elements $\sigma_{i j}=\delta V / \delta h_{i j}$, $\underline{\pi}$ is second order tensor.

We have used the Nose-Hoover formulation in the present study. $^{25}$

\section{Intermolecular potential}

Malonic acid has been modeled using short range Lennard-Jones and long-range Coulombic interaction. The short range interaction parameters have been taken from Gromacs forcefield. Earlier, these parameters have been used for the simulation of the liquid phase of malonic acid. ${ }^{26}$ Their computed results showed good agreement with experimental data for many of the thermodynamic properties. Methyl $\left(\mathrm{CH}_{2}\right)$ group has been modeled as a united atom. In the Gromacs forcefield several of the bond types are modeled with the help of harmonic potentials and these are listed in Table 1 . Similarly each molecule of malonic acid is modeled in terms of five distinct bond angles and four dihedral angles and one improper dihedral angle. These are also listed in Table 1. The short range non-bonded interaction terms are also tabulated. For the charge on various atoms we have investigated two different models: CHELPG and MAL. The charges for these two models are listed along with short range parameters. In the CHELPG model the charges are derived based on fit to molecular electrostatic potential field while the MAL model is based on the Mulliken charges obtained from first principle density functional theory calculations (B3LYP/6-311G++(d,p)). Molecular dynamics simulation employing the variable shape ParrinelloRahman simulation cell for CHELPG and MAL were carried out. The results of these two models are listed in Table 2. CHELPG predicts lattice parameters a, b, c in reasonable agreement with experimental value at $300 \mathrm{~K}$. However the agreement between $\alpha, \beta$ and $\gamma$ obtained for CHELPG compares poorly with the experimental values. For example, the value for $\alpha$ is 100.6 which compares poorly with the experimental value of 108.5. Similarly $\beta$ and $\gamma$ deviates more than 5\%. MAL on the other hand shows reasonable agreement in all the lattice parameters. The maximum deviation of $5 \%$ is seen for $c$. We have therefore employed MAL for the rest 
Table 1. Intermolecular interacion potential parameters employed in the molecular dynamics simulation of malonic acid.

\begin{tabular}{|c|c|c|c|c|}
\hline Bond Type $^{a}$ & $\mathrm{k}(\mathrm{kcal} / \mathrm{mol})$ & \multicolumn{3}{|c|}{$\mathrm{r}_{0}$} \\
\hline $\begin{array}{l}\mathrm{H}_{O H}-\mathrm{O}_{O H} \\
\mathrm{O}_{O H}-\mathrm{C}_{C=O} \\
\mathrm{C}_{C=O}-\mathrm{O}_{C=O} \\
\mathrm{C}_{C=O}-\mathrm{CH}_{2}\end{array}$ & $\begin{array}{c}750.0 \\
900.0 \\
1200.0 \\
800.0\end{array}$ & & $\begin{array}{l}1.00 \\
1.36 \\
1.23 \\
1.53 \\
\end{array}$ & \\
\hline Angle Type ${ }^{a}$ & $\mathrm{k}(\mathrm{kcal} / \mathrm{mol})$ & \multicolumn{3}{|c|}{$\theta_{0}$} \\
\hline $\begin{array}{l}\mathrm{H}_{O H}-\mathrm{O}_{O H}-\mathrm{C} \\
\mathrm{O}_{O H}-\mathrm{C}_{C=O}-\mathrm{O}_{C=O} \\
\mathrm{O}_{C=O}-\mathrm{C}_{C=O}-\mathrm{CH}_{2} \\
\mathrm{C}_{C=O}-\mathrm{CH}_{2}-\mathrm{C}_{C=O} \\
\mathrm{O}_{O H}-\mathrm{C}_{C=O}-\mathrm{CH}_{2}\end{array}$ & $\begin{array}{c}95.0 \\
120.0 \\
120.0 \\
110.0 \\
120.0\end{array}$ & \multicolumn{3}{|c|}{$\begin{array}{l}109.5 \\
124.0 \\
121.0 \\
111.0 \\
115.0\end{array}$} \\
\hline Dihedral Type ${ }^{b}$ & $\mathrm{k}(\mathrm{kcal} / \mathrm{mol}) / \mathrm{rad}^{2}$ & $\mathrm{n}$ & \multicolumn{2}{|r|}{$\mathrm{d}$} \\
\hline $\begin{array}{l}\mathrm{H}_{O H}-\mathrm{O}_{O H}-\mathrm{C}_{C=O}-\mathrm{O}_{C=O} \\
\mathrm{H}_{O H}-\mathrm{O}_{O H}-\mathrm{C}_{C=O}-\mathrm{CH}_{2} \\
\mathrm{O}_{C=O}-\mathrm{C}_{C=O}-\mathrm{CH}_{2}-\mathrm{C}_{C=O} \\
\mathrm{O}_{O H}-\mathrm{C}_{C=O}-\mathrm{CH}_{2}-C_{C=O} \\
\mathrm{C}_{C=O}-\mathrm{O}_{O H}-\mathrm{CH}_{2}-\mathrm{O}_{C=O}^{d}\end{array}$ & $\begin{array}{c}4.0 \\
4.0 \\
0.1 \\
0.1 \\
80.0\end{array}$ & $\begin{array}{l}2 \\
2 \\
6 \\
6\end{array}$ & \multicolumn{2}{|c|}{$\begin{array}{c}180 \\
180 \\
0 \\
0 \\
0\left(\chi_{0}\right)\end{array}$} \\
\hline Atom Type & $\sigma(\AA)$ & $\epsilon(\mathrm{kcal} / \mathrm{mol})$ & $\mathrm{MAL}^{c}$ & CHELPC \\
\hline $\begin{array}{l}\mathrm{H}_{O H} \\
\mathrm{O}_{O H} \\
\mathrm{C}_{C=O} \\
\mathrm{O}_{C=O} \\
\mathrm{CH}_{2}\end{array}$ & $\begin{array}{c}0.0 \\
2.955 \\
3.361 \\
2.626 \\
3.9647\end{array}$ & $\begin{array}{c}0.0 \\
0.2029 \\
0.0970 \\
0.4122 \\
0.1400\end{array}$ & $\begin{array}{c}0.29 \\
-0.18 \\
0.02 \\
-0.25 \\
0.24\end{array}$ & $\begin{array}{c}0.44 \\
-0.64 \\
0.78 \\
-0.56 \\
-0.04\end{array}$ \\
\hline
\end{tabular}

${ }^{a} E=k\left(x-x_{0}\right)^{2} ;{ }^{b} E=k(1+\cos (n \phi-d))$

${ }^{c}$ MAL partial charges from B3LYP/6-311G* DFT calculations

$d$ improper angle of the form $k\left(\chi-\chi_{0}\right)^{2}$

of the simulation and results from this model alone is presented below.

\section{Computational methods}

All molecular dynamics simulations have been carried out in NPT ensemble with variable simulation cell using DLPOLY classic version. ${ }^{27}$ Malonic acid crystallizes P1 group. One unit cell of malonic acid has two molecules. A simulation cell was constructed with $6 \times 6 \times 4$ unit cells with a total of 288 molecules. The $\mathrm{CH}_{2}$ group is modeled with united atom with the mass of 14.02 a. $u$. All long range interactions were computed with the help of Ewald sum. Periodic boundary conditions were imposed along the three directions. An integration time step of $1 \mathrm{fs}$ has been used. Conservation in energy was better than 1 in $10^{4}$. Equilibration was carried out for $500 \mathrm{ps}$, which was followed by a production run of $4 \mathrm{~ns}$. For the purpose of calculations of various properties, positions, velocities and forces were stored every $10 \mathrm{fs}$ over 4 ns duration.

\subsection{Calculation of elastic constants}

All the elastic constants were estimated with the help of DLPOLY program. From Hooke's law the elastic moduli can be expressed in terms of the equation

$$
\sigma_{i j}=C_{i j k l} \epsilon_{k l}
$$

$\sigma$ is the stress tensor and $\epsilon$ is strain tensor. As the linear relation between the strain and stress is valid only for small values of strain, we have computed the elastic constants with small values of strain. In general, there should be $3^{4}$ or 81 components of second-rank tensor $\mathrm{C}_{i j k l}$. Of these, only 36 are independent elastic constants. However, since the $6 \times 6$ matrix is symmetric, there are just 21 elastic constants.

To determine these components, we have modified the h-matrix by introducing a strain:

$$
h=(1-e) h_{0}
$$

where $h_{0}$ is the undistorted simulation cell h-matrix and $e$ is the induced strain. The resulting matrix is $h$. Using 
the strained simulation cell or $h$, a $N V T$ run was carried out at $300 \mathrm{~K}$. This run was equilibrated for $50 \mathrm{ps}$ and production run was carried out for another 50 ps. The average stress tensor during the production run was computed to obtain all the elastic constants from the slope of the stress versus strain plot.

This method is usually referred as direct method of evaluation, and has been used before as reported by Guo et al. ${ }^{28,29}$

\section{Results and Discussion}

\subsection{Structure and energetics}

Before we compute properties of the hydrogen bond chains and the elastic properties of malonic acid crystal, it is necessary to establish that the intermolecular potential we employ is able to predict accurately the crystal structure and enthalpy. Towards this, simulations have been carried out starting with X-ray crystallographic structure of the triclinic phase of malonic acid $(\mathrm{P} \overline{1}) .{ }^{18,30}$ Parrinello-Rahman method permits the lattice parameters to evolve and change to any of the seven crystal systems. The variation during the course of the simulation of the lattice parameters $a, b, c, \alpha, \beta$ and $\gamma$ for the triclinic phase are shown in Figure 1. The average lattice parameters obtained from the MD simulation are listed in Table 2, at three different temperatures along with the heat of sublimation. Experimental values of the lattice parameters at 130 and $300 \mathrm{~K}$ are also listed in the Table 2. The potential(CHELPG) (ref. Table 1) based on a fit to the electrostatic potential field yields reasonable agreement of the lattice parameters $a, b$ and $c$. However, agreement in $\alpha, \beta$, and $\gamma$ is very poor. Further, $\alpha$ and $\gamma$ are rather close to each other suggesting that the converged crystal structure does not belong to triclinc crystal system. On the other hand the model MAL potential (ref. Table 2) provides reasonable agreement in all the lattice parameters $(a, b, c, \alpha, \beta, \gamma)$, Therefore, below we report results for only the MAL potential. Although there are reports of other phases of malonic acid ${ }^{31}$ the detailed structure of these phase are not available.

Malonic acid has the following distinct atom types (where we indicate in parenthesis, the chemical group to which the atom belongs): (i) $\mathrm{H}_{O H}$ (ii) $\mathrm{O}_{O H}$ (iii) $\mathrm{C}_{C O}$ (iv) $\mathrm{O}_{\mathrm{CO}}$ and (v) $\mathrm{CH}_{2}$. Thus, there are in all five distinct types of atoms. These yield 15 different RDFs. These RDFs have been divided into five distinct groups, based on the five groups stated above. Here, we represent $\mathrm{CH}_{2}$ as an united atom since this simplifies the calculation.
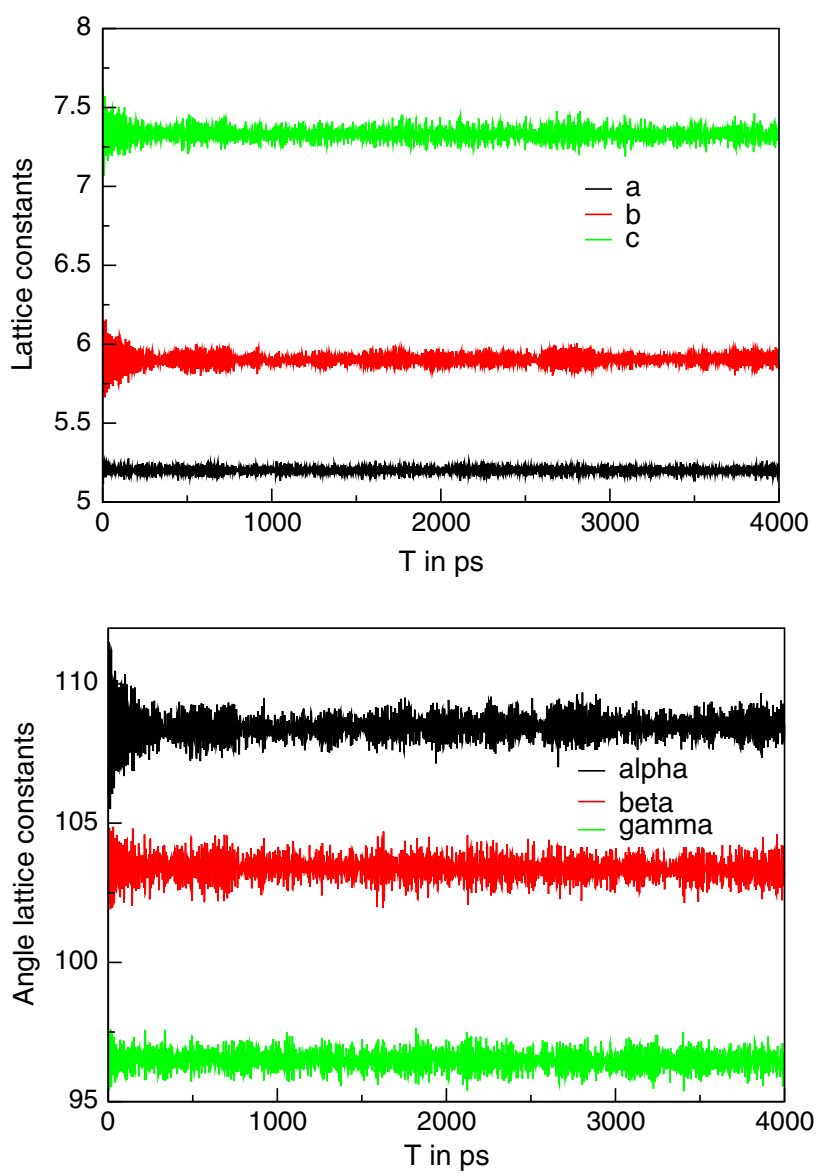

Figure 1. Time evolution of lattice parameters of malonic acid triclinic phase $\mathrm{P} \overline{1}$.

Radial distributions functions(RDFs) between these are shown in Figure 2.

The $\mathrm{H}_{O H}-\mathrm{O}_{C O} \mathrm{RDF}$ has a peak at $1.78 \AA$ which is shorter than $\mathrm{H}_{O H}-\mathrm{X} \mathrm{RDF}\left(\mathrm{X}=\mathrm{O}_{O H}, \mathrm{H}_{O H}, \mathrm{C}_{C O}, \mathrm{CH}_{2}\right)$. In contrast, all the other RDFs involving $\mathrm{H}_{O H}$ exhibit a first peak in the range $\sim 2.7-3.9 \AA$. The short contact between $\mathrm{H}_{O H}-\mathrm{O}_{C O}$ is attributed to the hydrogen bonding between $\mathrm{H}_{O H}$ of one molecule and $\mathrm{O}_{C O}$ of the neighbouring molecule. Similar short contact $(\sim 2.8 \AA)$ is also seen between $\mathrm{O}_{O H}$ and $\mathrm{O}_{C O}$ of the neighbouring molecule which is shorter than the average $\sim 3.6 \AA$. The figure also shows that the main hydrogen bond is between $\mathrm{H}_{O H}$ and $\mathrm{O}_{C O}$ and not with $\mathrm{H}_{O H}$. This suggests the intermolecular potential employed here does reproduce the chemical nature of malonic acid well in spite of its purely classical description. The $\mathrm{CH}_{2}-\mathrm{CH}_{2}$ RDF exhibits a broad peak between 4.0 and $5.0 \AA$ A with a shoulder between 5.5 and $6.0 \AA$. The $\mathrm{CH}_{2}$ group may be said to represents the c.o.m. of the molecule. The first peak is broad suggesting that the c.o.m. of the molecule has a large degree of freedom relative to the other atoms of the molecule. Ma et al., have investigated malonic acid in water and they find a broad peak between 4.0 and 
Table 2. Average lattice constants of the triclinic phase $\mathrm{P} \overline{1}$ of malonic acid at different temperatures obtained from molecular dynamics simulation. Also listed are the experimental values. MAL predictions are in better agreement with experiment. Errors in the computed values of lattice parameters are less than $\pm 0.1 \AA$.

\begin{tabular}{|c|c|c|c|c|c|c|}
\hline \multirow[t]{2}{*}{ Lattice Constants } & \multirow[t]{2}{*}{ CHELPG(300K) } & \multicolumn{3}{|l|}{$\underline{\text { MAL }}$} & \multicolumn{2}{|l|}{ Expt } \\
\hline & & $300 \mathrm{~K}$ & $250 \mathrm{~K}$ & $130 \mathrm{~K}$ & $130 \mathrm{~K}$ & $300 \mathrm{~K}$ \\
\hline a $(\AA)$ & $5.14(0.47)$ & $5.05(2.20)$ & 5.05 & $5.05(2.13)$ & 5.16 & 5.16 \\
\hline $\mathrm{b}(\AA)$ & $5.18(3.15)$ & $5.65(5.65)$ & 5.59 & $5.55(4.12)$ & 5.33 & 5.35 \\
\hline$c(\AA)$ & $8.06(4.22)$ & $8.00(5.01)$ & 7.82 & $7.72(5.85)$ & 8.20 & 8.42 \\
\hline$\alpha$ & $100.65(7.25)$ & $106.18(2.15)$ & 106.62 & $105.96(1.95)$ & 108.07 & 108.52 \\
\hline$\beta$ & $96.87(4.66)$ & $102.75(1.13)$ & 102.77 & $102.50(1.24)$ & 101.24 & 101.60 \\
\hline$\gamma$ & $101.81(6.11)$ & $93.44(3.66)$ & 97.99 & $98.67(3.57)$ & 95.27 & 95.95 \\
\hline Volume $\left(\AA^{3}\right)$ & $203.27(4.94)$ & $207.55(2.94)$ & 201.55 & $198.15(4.33)$ & 207.11 & 213.84 \\
\hline
\end{tabular}
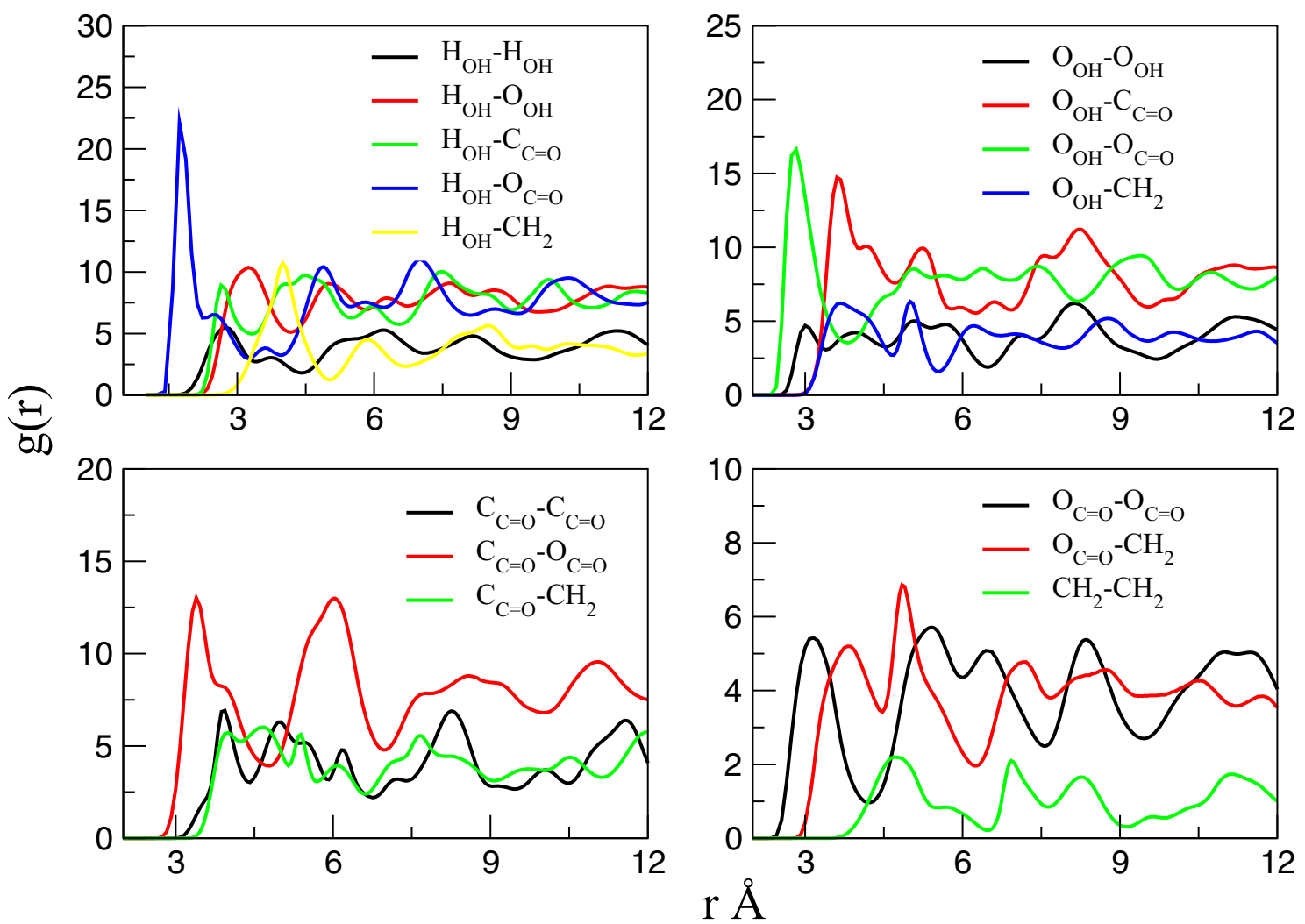

Figure 2. RDFs at $300 \mathrm{~K}$ between different atoms or groups of malonic acid in the triclinic phase $\mathrm{P} \overline{1}$. There are in all 15 RDFs.

6.0 A without an explicit shoulder. ${ }^{26}$ The fact that $\mathrm{CH}_{2}$ $\mathrm{CH}_{2} \mathrm{RDF}$ in the crystal obtained by us here has a full width at half maximum that is relatively large for a crystal suggests significant motion of malonic acid c.o.m. within the crystal. The RDFs exhibit distinct peaks as one would expect from a well-ordered crystal.

Finally, we show the temperature dependent RDF between $\mathrm{CH}_{2}-\mathrm{CH}_{2}$ groups in Figure 3. By about 300 $\mathrm{K}$, various peaks in RDF begin to merge and the fine structure disappears. Enthalpy of sublimation of malonic acid from experiment is $108.9 \mathrm{~kJ} / \mathrm{mol}(\mathrm{T}=339-357$ $\mathrm{K}) .{ }^{14}$ The enthalpy of sublimation is given by

$\Delta H_{\text {sub }}=\Delta E_{\text {therm }(s)}+\Delta E_{s \rightarrow l}+\Delta E_{\text {therm }(l)}+\Delta E_{l \rightarrow g}$

where $\Delta E_{\text {therm }}(s)=\int_{0}^{T_{m}} C_{p}(s) d T, \Delta E_{\text {therm }}(l) \stackrel{(}{=}$ $\int_{T_{m}}^{T^{b}} C_{p}(l) d T ; \Delta E_{s \rightarrow l}$ and $\Delta E_{l \rightarrow g}$ are the changes in total energy on going from solid to liquid and liquid to 


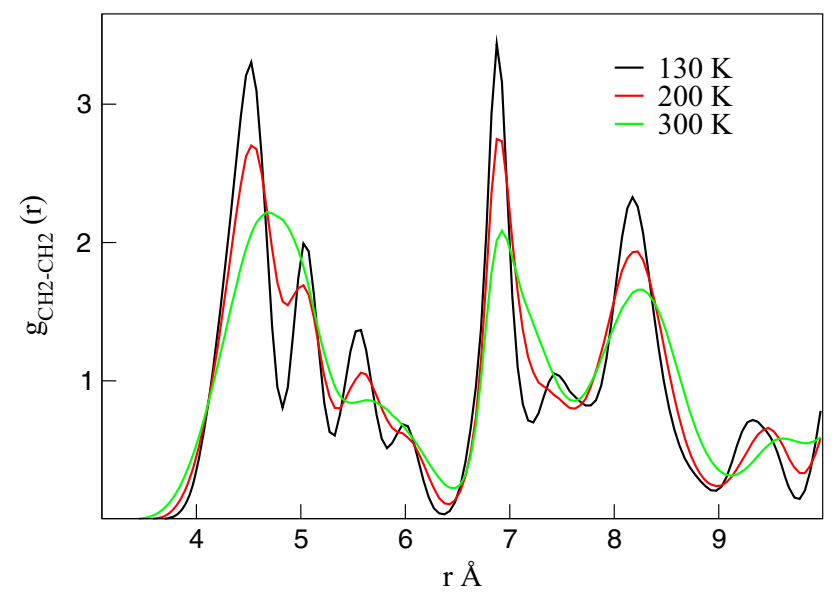

Figure 3. RDFs between methylene groups in neighbouring molecules in the triclinic crystalline phase of malonic acid.

gas respectively. In the present study we have estimated $\Delta H_{\text {sub }}$ from

$$
\Delta H_{s u b}=E_{g}^{p o t}(T)-E_{s}^{p o t}(T)+R T
$$

Similarly enthalpy of vaporization is given by

$$
\Delta H_{v a p}=E_{g}^{p o t}(T)-E_{l}^{p o t}(T)+R T
$$

where $E_{x}^{p o t}(T)$ is the potential energy of interactions in the $\mathrm{x}$ phase,where $\mathrm{x}$ is solid, liquid or gas and $\mathrm{RT}$ is the $p\left(V_{g}-V_{l}\right)$ term where $V_{l}$ is negligible compared to $V_{g}$.

The computed value for the enthalpy of sublimation is $99.75 \mathrm{~kJ} / \mathrm{mol}$ which is about $8 \%$ lower than the experimental value. The calculated enthalpy of vaporization is $75.43 \mathrm{~kJ} / \mathrm{mol}$, Goddard and coworkers have reported values for enthalpy of vaporization as $68.18 \pm 6.11 \mathrm{~kJ} / \mathrm{mol}$ at $580 \mathrm{~K} .^{32}$

Eight dihedral angles are possible in a single malonic acid molecule. They are reduced into three distinct types with relevant force field parameters to model the torsional energies (see Table 1). Figure 4 shows dihedral distribution for all the dihedral angles obtained from MD.

From the above properties, it is evident that the intermolecular potential is able to provide a realistic description of the malonic acid crystal. We now look at the hydrogen bonds and chains of hydrogen bonds in the crystal. To the best of our knowledge, no previous attempt at characterizing the chains of hydrogen bonds in such solids exist in the literature.

\subsection{Hydrogen bond analysis}

As we already saw in the introduction, there have been investigations which suggest that the elastic constants can be high in a crystal along certain directions and that

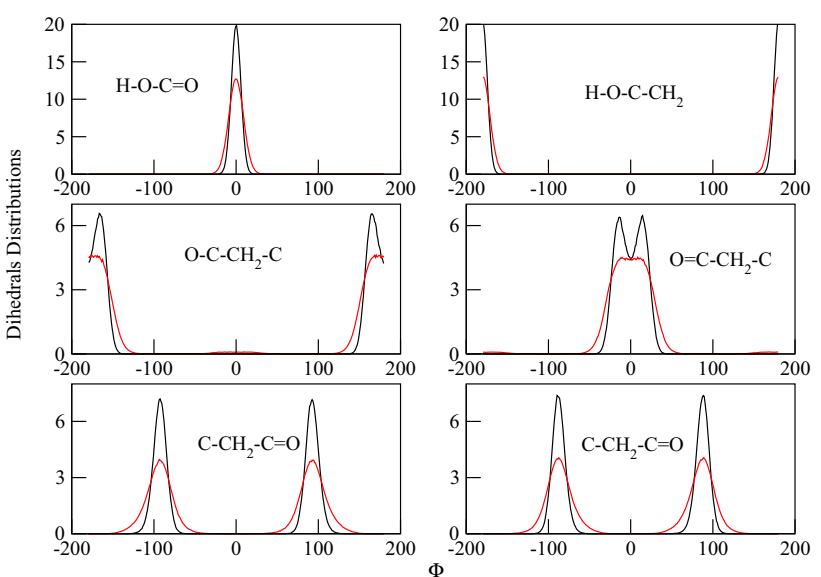

Figure 4. Dihedral distribution for the distinct angles (as shown in the legend) black corresponds to $130 \mathrm{~K}$ and red for $300 \mathrm{~K}$.

these could be due to presence of hydrogen bond chains along those directions. ${ }^{1}$

Carboxylic acid functional groups have a significant ability to form strong hydrogen bonds. Jefferey et al. have classified the type of hydrogen bond X-H...A (where $\mathrm{X}$ is the donor and $\mathrm{A}$ is the acceptor) according to directionality, angles, $\mathrm{X}-\mathrm{H}$ lengthening, etc. The hydrogen bond is said to be strong if it satisfies the following criteria ${ }^{33}$ :

$$
\begin{aligned}
& 2.2<\mathrm{X} \ldots \mathrm{A}<2.5 \AA, 170^{\circ}<\angle \mathrm{XHA}<180^{\circ}, \\
& 1.2<\mathrm{H} \ldots \mathrm{A}<1.5 \AA
\end{aligned}
$$

From Jeffrey's definition hydrogen bond between two malonic acid molecules may be classified as moderate to strong.

Luzar and Chandler and several others have investigated the hydrogen bond dynamics in liquid water. ${ }^{34,35}$ Ohmine and co-workers have investigated the reported average lifetime of hydrogen bond of liquid water and ice. ${ }^{36}$ To the best of our knowledge, there are no studies of hydrogen bond dynamics in non-aqueous crystalline systems. These can be obtained by defining the hydrogen bond status variable $h(t)$ which is unity if two molecules under consideration are hydrogen bonded at $t$ and zero if there is no hydrogen bond between them. In the case of malonic acid, based on the computed distributions of distances and angles, the following criteria are used to determine if two neighboring molecules are hydrogen bonded or not:

$$
\mathrm{H} \ldots \mathrm{A} \leq 2.5 \AA, \angle \mathrm{AHX} \leq 30.0^{\circ}, \mathrm{X} \ldots \mathrm{A} \leq 3.9 \AA
$$

The distance of $3.9 \AA$ is the first minimum of the X...A RDF. We note that the RDF is averaged over all directions and not just [011] along which hydrogen bond chains exist. Therefore, the minimum could be lower 
for [011] direction. However, the choice of $3.5 \AA$ or 3.9 $\AA$ might not change the results here significantly since both are likely to give similar curves for the correlation functions reported below. The choice of the forcefield could also play an important role here. We have computed the intermittent hydrogen bonds $C_{h b}(t)$ where:

$$
C_{h b}(t)=<h(0) h(t)>/<h(0) h(0)>
$$

The continuous hydrogen bond correlation function, $S_{h b}(t)$ is defined by:

$$
S_{h b}(t)=<h(0) H(t)>/<h(0) h(0)>
$$

The former $\left(C_{h b}(t)\right)$ permits hydrogen bond to be reformed after the bond is broken at intermediate times whereas the continuous hydrogen bond correlation function does not permit such re-formation. Continuous hydrogen bond correlation function is shown in Figure 5.

In malonic acid crystal, the intermittent correlation function $C_{h b}(t)$ is found to decay extremely slowly. This is understandable since in the solid phase there is no long-range diffusion of molecules, and therefore neighbours remain the same and consequently there is no lasting severance of the hydrogen bond leading to no decay of the $C_{h b}(t)$. We have plotted $\ln \left(S_{h b}(t)\right)$ as a function of time in Figure 5. These have been averaged over all hydrogen bonds in the simulation cell, irrespective of the direction of the hydrogen bond (see below for the direction dependent hydrogen bond analysis). A linear fit to the plot gave a value of $24.1 \mathrm{ps}$ for the relaxation time of $\tau$ (at $\mathrm{T}=300 \mathrm{~K}$ ). These values in the solid phase are expected to be much larger than the values for the liquid phase. Chandra has reported 0.5 ps as the relaxation time in a dilute aqueous solution of $\mathrm{NaCl} .{ }^{35}$ Similar studies by using hydrogen bond correlation function for

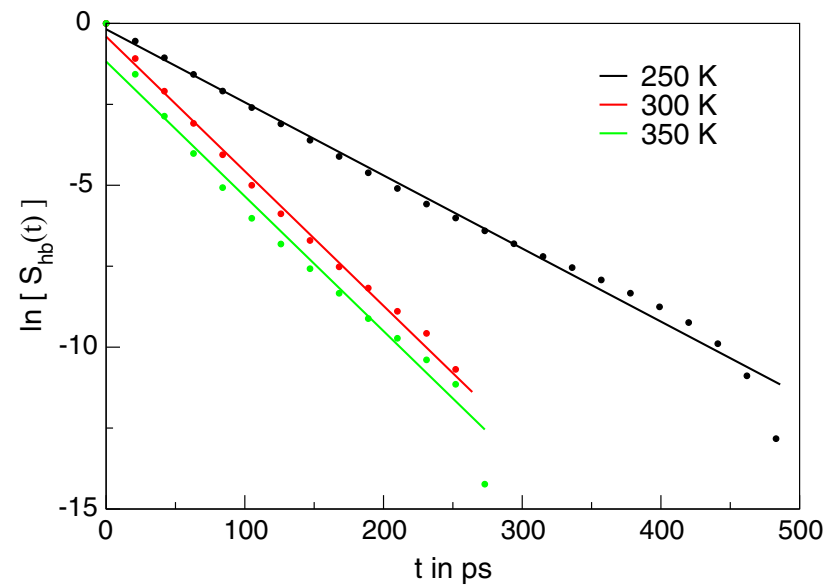

Figure 5. Continuous correlation function $\mathrm{S}_{h b}(\mathrm{t})$ for the hydrogen bond for different temperature.

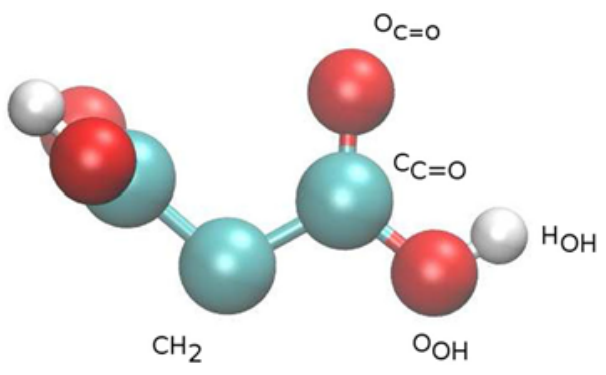

Figure 6. A representation of the initial configuration of the malonic acid molecule as found in the crystal from X-diffraction. Note the different orientations of the $-\mathrm{COOH}$ groups. The plane containing $\mathrm{O}_{C=O}, \mathrm{C}_{C=O}$, and $\mathrm{O}_{O H}$ atoms of one carboxylic group is nearly perpendicular to the $\mathrm{O}_{C=O}, \mathrm{C}_{C=O}$, and $\mathrm{O}_{O H}$ atoms of the other carboxylic group.

analysis of aqueous phase can be seen in Ref. ${ }^{37,38}$ Earlier in an interesting study of nucleation and growth of ice Ohmine and co-workers have reported average lifetime of hydrogen bond of liquid water at $300 \mathrm{~K}$ as 1.0 ps and of ice as $180 \mathrm{ps}$ at $230 \mathrm{~K} .{ }^{36}$ Our values obtained here therefore appear reasonable. The value of $24.1 \mathrm{ps}$ is less than that found in ice. Our calculations provide an estimate of the lifetime of hydrogen bond in organic crystals such as dicarboxylic acid crystals (Figure 6).

Hydrogen bond chains along [011] crystallographic direction: Unlike in a liquid, directionality is important in a crystal particularly in malonic acid which crystallizes in a triclinic space group. Further, an analysis of the lifetime of the hydrogen bond chains is likely to be important for many processes. An example of such a process is proton conduction. ${ }^{8}$ In Figure 7 we show a snapshot of the crystal looking down $\mathrm{X}$-axis. It is seen that hydrogen bonds exist between neighbouring molecules predominantly along certain crystallographic directions. In the malonic acid crystal these are [011] direction. For purposes of clarity, in Figure 8 we have plotted the same view by showing only the center of mass (c.o.m) by small spheres and the hydrogen bond by lines. Thus, two neighboring molecules are connected by a line if there exists a hydrogen bond between them. Two neighbouring malonic acid molecules have two $-\mathrm{COOH}$ groups facing each other and therefore, they could form two hydrogen bonds ((1)CO...HO(2) and (1)OH...CO(2) where 1 and 2 refer to molecules 1 and 2). In the analysis reported below, molecules 1 and 2 are said to have formed a hydrogen bond even if one of these bonds are formed as per the criteria stated earlier, namely, H . . A $\leq 2.5 \AA, \angle \mathrm{AHX} \leq 30.0^{\circ}$, $\mathrm{X} \ldots \mathrm{A} \leq 3.9 \AA$. Finally, note that in Figure 8 , apart 


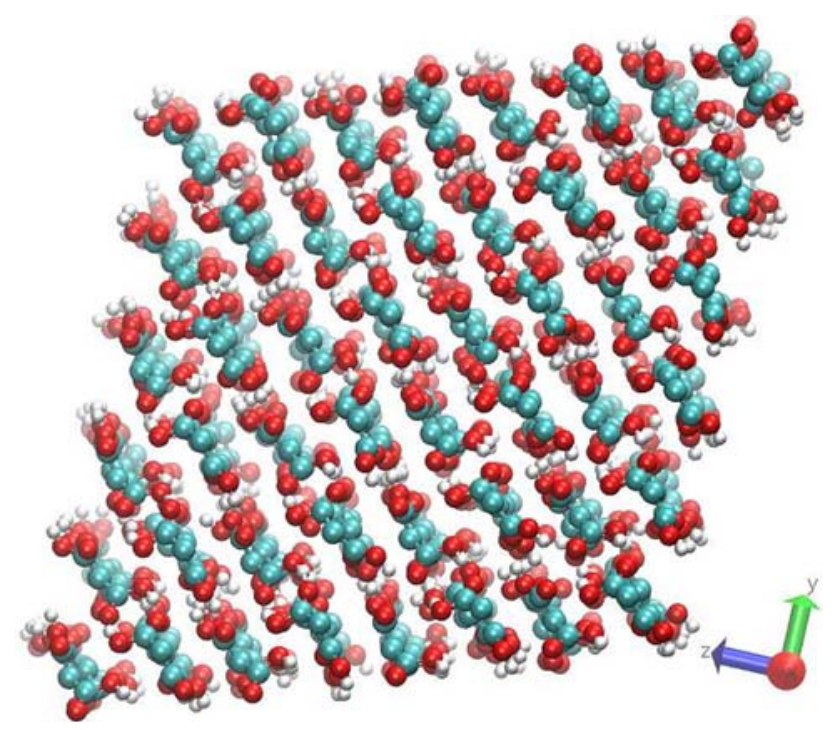

Figure 7. A snapshot of the $6 \times 6 \times 4$ unit cells looking down the $\mathrm{X}$-axis. The hydrogen bond chains are along [011] direction.

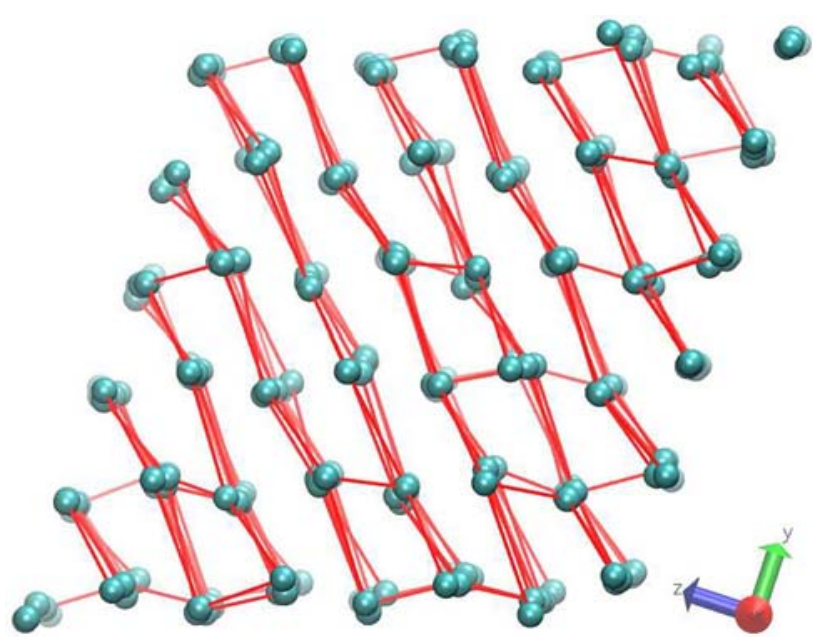

Figure 8. Cyan dots represent centre of mass of malonic acid molecule and red lines indicate hydrogen bond between them - the bonds are clearly along the [011] direction. Hydrogen bonds between two linear chains of the malonic acid molecule which are usually very short-lived can also be seen. The intrachain bonds have long lifetimes and therefore are important in determining the properties of the solid phase.

from intrachain hydrogen bonds, there are also interchain hydrogen bonds. These are, however, very short lived and therefore do not contribute much to any property of the solid. For this reason we have not included these interchain hydrogen bonds in the calculation of the continuous hydrogen bonds correlation function, $S_{h b}(t)$. Also, these hydrogen bonds are not along [011] direction and the criteria we describe below are not satisfied by the interchain hydrogen bonds.
Note that the hydrogen bonded molecules form a zigzag chain. These chains make alternately an angle in the range 34-37 degrees or 149-151 degrees with the [011] direction. Hence, an additional condition of the angle the bond makes with [011] direction is imposed to select only the hydrogen bonds along the zig-zag chain but around the [011] direction.

From single hydrogen bond correlation function we now wish to compute the properties of chains of hydrogen bonds of different lengths. But we still do not have a definition for the continuous hydrogen bond correlation function for a chain. We indicate the length of the hydrogen bond chain by $l$. The bond between a pair of molecules corresponds to $l=1$, that is, chain of length unity. $l=2$ corresponds to three molecules participating in hydrogen bond formation between themselves and their neighbours just the way it is computed for $l$ $=1$. However, the calculation of $h(t)$ is confined to just the two bonds under consideration. Note that we are considering here only linear chains. Here molecule 1 is hydrogen bonded to 2 and 2 to 3 . Thus, a chain of length $l$ will have hydrogen bond between $l+1$ molecules. The $C_{h b}^{l}$ and $S_{h b}^{l}$ for the chain is defined as the

$$
C_{h b}^{l}(t)=\left\langle h^{l}(0) \cdot h^{l}(t)\right\rangle /\left\langle h^{l}(0) \cdot h^{l}(0)\right\rangle
$$

where

$$
\begin{aligned}
h^{l}(t) & =\Pi_{j=1}^{l} h_{j}(t) \\
H^{l}(t) & =\Pi_{j=1}^{l} H_{j}(t) \\
S_{h b}(t) & =<h^{l}(0) H^{l}(t)>/<h^{l}(0) H^{l}(0)>
\end{aligned}
$$

The Heaviside functions $h^{l}(t)$ is unity if and only if $h(t)$ for each of the hydrogen bonds in the linear chain exist at that moment. Even if one of the hydrogen bonds in the link is not formed then $\mathrm{h}(\mathrm{t})$ becomes zero. Similar definition applies to $H^{l}(t)$. Note that two neighbouring molecules of malonic acid can form hydrogen bonds through their $\mathrm{OH}$ as well as $\mathrm{CO}$ groups. Often, two neighbouring molecules are bonded with more than one hydrogen bond.

The continuous hydrogen bond correlation function $S_{h b}(t)$ in a crystal, however, decays faster since the vibrational motion around the equilibrium position around the c.o.m leads to a snapping of the hydrogen bond between two neighbours at certain moments. This leads to break in continuity necessary for decay of $S_{h b}(t)$. A plot of $\ln \left(S_{h b}(t)\right)$ against time is shown in Figure 9 for different $l$. It is seen that decay of these functions is faster with increase in chain length $l$.

The values of $\tau$ for various $l$ are listed in Table 3 . These values show a decrease in $\tau$ with increase in $l$ and are plotted in Figure 9. The values of $\tau$ are around $500 \mathrm{ps}$ at $250 \mathrm{~K}$ for $l=1$ but decrease to around 100 

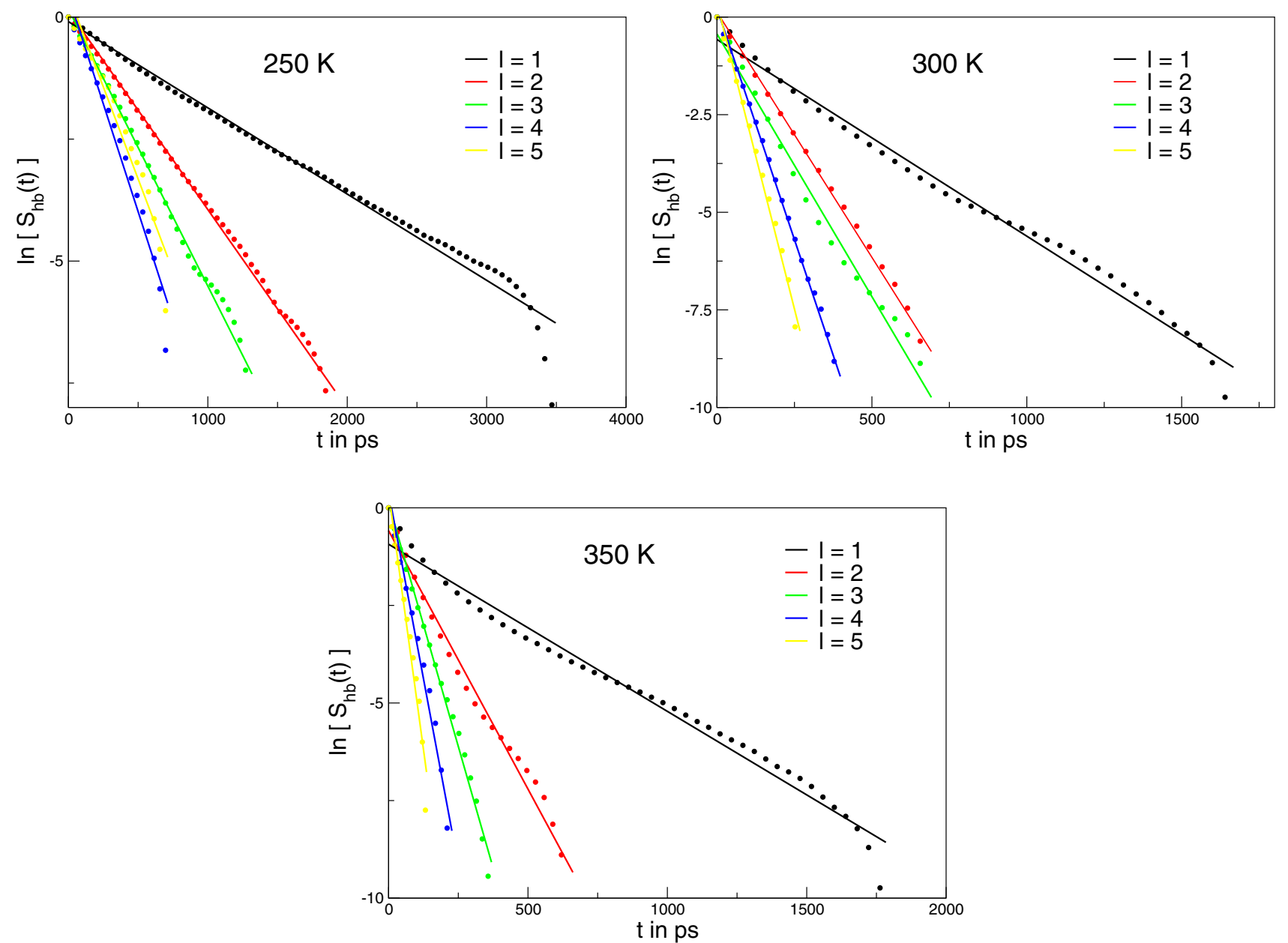

Figure 9. $S_{h b}(t)$ for the linear chain along [011] direction at different temperatures.

Table 3. Hydrogen bond relaxation time as a function of length of the chain, $l$, at three different temperatures in the direction [011] in $\mathrm{P} \overline{1}$ phase of malonic acid.

\begin{tabular}{rrrr}
\hline$l$ & $250 \mathrm{~K}$ & $\begin{array}{c}300 \mathrm{~K} \\
p s\end{array}$ & $350 \mathrm{~K}$ \\
\hline 1 & 566.0 & 198.6 & 233.6 \\
2 & 245.5 & 79.6 & 75.5 \\
3 & 176.1 & 74.4 & 40.1 \\
4 & 113.1 & 42.2 & 26.2 \\
5 & 132.4 & 31.9 & 19.0 \\
\hline
\end{tabular}

ps for $l=5$. The longer lifetimes can lead to more facile proton conduction from one molecule to the fifth neighbouring molecule but the activation energies for proton hopping may not permit easy hopping from one molecule to another at low temperatures. By $350 \mathrm{~K}$ the value of the lifetime of a chain of length 5 is around 18 ps. 5.2a Temperature dependence of hydrogen bond relaxation time: In Figure 9, continuous hydrogen bond correlation function $S_{h b}(t)$ at $300 \mathrm{~K}$ is shown. The time evolution of $S_{h b}(t)$ is shown for approximately 2 ns for $l=1$ to 5 . For $l=1$, the function decays slowly with a relaxation time of 198 ps obtained from the slope of the straight line fit to $\ln \left(S_{h b}(t)\right)$ vs. time plot. At higher $l$ values, the relaxation time gradually decrease: 79.6, $74.4,42.2$ and 31.9 ps for $l=2,3,4$ and 5 respectively at $300 \mathrm{~K}$. We have also computed the relaxation times at other temperatures. At $250 \mathrm{~K}$, the values are higher. Also, in general the relaxation times are lower, higher the value of $l$. Note that there are some anomalies seen in the data which may be due to the limited accuracy of the computed relaxation times.

We have plotted $\ln \left(\tau_{1}\right)$ against reciprocal temperature in Figure 10. The relevant Arrhenius expression is $\tau_{1}=\tau_{0}$ $\exp \left(-E_{a} / R T\right)$ where $\tau_{0}$ is the pre-exponential constant and $E_{a}$ is the activation energy. $\mathrm{R}$ is the gas constant. We have obtained activation energies from the slope of the fitted line. These are listed in Table 4 It is seen that 


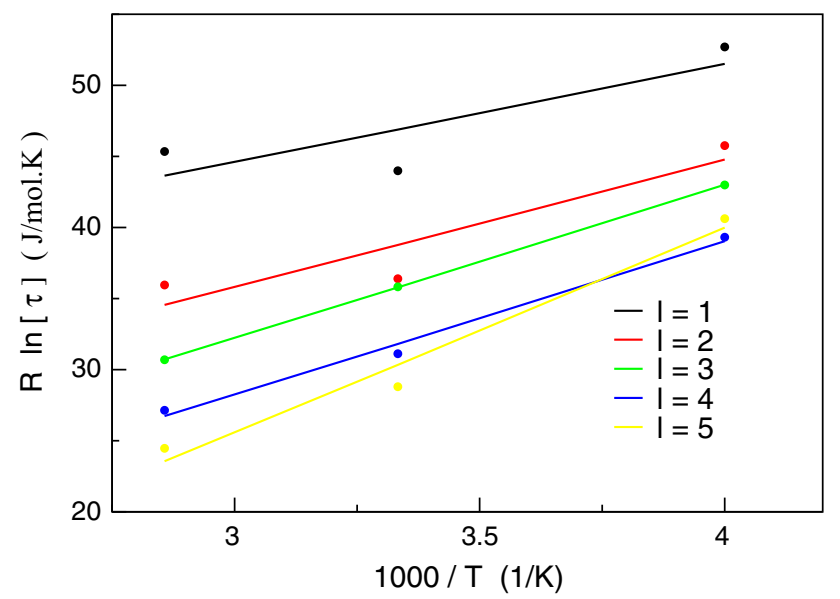

Figure 10. A plot of $\mathrm{R} \ln \tau$ vs $1 / \mathrm{T}$ for linear chains of differing lengths $l$.

the activation energies increase with $l$. The $E_{a}$ values increase from $6.9 \mathrm{~kJ} / \mathrm{mol}$ for $l=1$ to $14.4 \mathrm{~kJ} / \mathrm{mol}$ for $l$ $=5$. Note that the difference between the $E_{a}$ value for successive chain lengths or $l$ values is $1.8 \mathrm{~kJ} / \mathrm{mol}$ except between $l=1$ and 2 where the difference is $2 \mathrm{~kJ} / \mathrm{mol}$. There is, however, no increase in $E_{a}$ on going from $l$ $=3$ to 4 . There is some anomaly at $l=4$ which needs more careful investigations. But on going from $l 3$ to 5 we see that the $E_{a}$ has increased from 10.78 to 14.41 $\mathrm{kJ} / \mathrm{mol}$ which is twice $1.8 \mathrm{~kJ} / \mathrm{mol}$. It appears that the anomalous increase in relaxation time of $l=5$ as compared to $l=4$ probably is related to the even number of malonic acids participating in the hydrogen bonding when $l=5$. For liquid water, the activation energies are higher than what is seen here: $9.8 \mathrm{~kJ} / \mathrm{mol}$ for bulk and 4.9 $\mathrm{kJ} / \mathrm{mol}$ for confined water. ${ }^{39,40}$ Note that bulk water has a three dimensional network of hydrogen bonds with each molecule of water having four hydrogen bonded neighbours. The present molecule has a predominantly one dimensional network of hydrogen bonds with two neighbours. Further, the hydrogen bonds in water are strong while those in malonic acid are of medium strength. Therefore, the value we have obtained here appears reasonable.

The dependence of activation energy on chain length can be understood in the following way. As the length of the chain increases, it decays (as obtained by intermittent and continuous hydrogen correlation functions) faster at a given temperature. At higher temperatures, the longer chains have lower lifetimes, lower than the shorter chains. In other words, the lifetime decreases more rapidly with temperature for longer chains than shorter chains. This leads to higher activation energies for longer chains. So, the results are to be expected and we do not think they are strange. The reason why $1=3$
Table 4. Activation energy $E_{a}$ obtained from the hydrogen bond relaxation times for various chain lengths $l$ obtained from (ref. Figure 10) the fit to the Arrhenius equation $\tau_{s}=\tau_{0} \exp \left(-\mathrm{E}_{a} / \mathrm{RT}\right)$.

\begin{tabular}{lc}
\hline$l$ & $E_{a}(\mathrm{~kJ} / \mathrm{mol})$ \\
\hline 1 & 6.899 \\
2 & 8.966 \\
3 & 10.780 \\
4 & 10.786 \\
5 & 14.409 \\
\hline
\end{tabular}

Table 5. Elastic tensor components for the triclinic phase of malonic acid. All 36 components are listed. However, the matrix should be symmetric. As the errors are not small, the elements $C_{i j k l}$ does not equal $C_{k l i j}$. There are, in fact, just 21 independent elastic constants.

\begin{tabular}{lrlrrrr}
\hline Components & xx & yy & \multicolumn{1}{c}{ zz } & \multicolumn{1}{c}{ yz } & xz & xy \\
\hline $\mathrm{xx}$ & 18.9 & 11.1 & 9.5 & 1.4 & 0.2 & 0.5 \\
yy & 10.4 & 22.8 & 15.2 & 12.7 & 3.4 & 2.3 \\
$\mathrm{zz}$ & 8.7 & 13.9 & 16.2 & 3.5 & 2.9 & 2.9 \\
$\mathrm{yz}$ & 1.6 & 3.5 & 2.6 & 3.8 & 1.0 & 0.1 \\
$\mathrm{xz}$ & 0.3 & 0.7 & 0.6 & 0.5 & 1.3 & 0.5 \\
$\mathrm{xy}$ & 0.5 & 0.6 & 0.7 & 0.1 & 0.4 & 0.8 \\
\hline
\end{tabular}

and $1=4$ have similar values might be due to limited length of the MD runs used to estimate these numbers. Longer MD runs might exhibit a more reliable dependence of activation energy on chain length.

\subsection{Elastic constants}

A triclinic structure has in all 36 elastic constants but only 21 of these are independent since the $6 \times 6$ tensor is symmetric. The computed actual $6 \times 6$ matrix is usually not symmetric because the elements elastic constants are of finite accuracy. Table 5 gives the 36 elastic constants.

These elastic constants have been obtained from molecular mechanics calculations by changing selectively the elements of the $h$ matrix defined earlier in the variable shape Parrinello-Rahman simulations. The stress tensor is computed at the strain induced by changing one of the elements of the $h$ matrix. Then a plot of stress against strain is made and from its slope we compute the corresponding elastic constant. We have then computed the $3 \times 3$ pressure tensor and from this we obtain the corresponding elastic constants (Figure 11).

All the constants in the Table 5 corresponds to secondrank tensors. Thus it should be read as $\mathrm{C}_{x x x x}$ for the first 


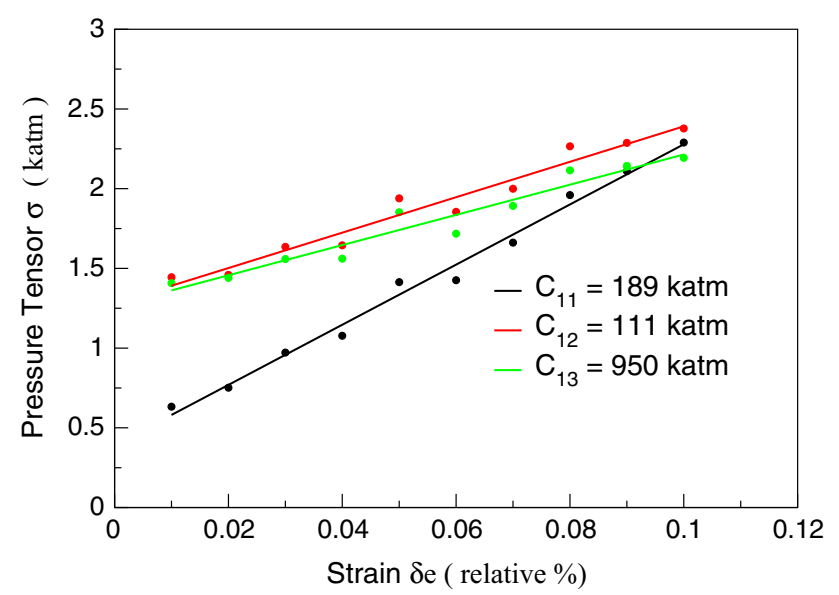

Figure 11. Representative linear fit for few elastic constants refer Table 5 .

row and first column element, which is elastic constant $\mathrm{C}_{11}$ in Voigt notation. As the pressure fluctuations are usually huge in typical $N V T$ ensemble, it is expected that measured stress tensors are prone to have relatively large error bars. The tabulated values represents slope from the linear fit for the equation $\sigma_{i j}=\mathrm{C}_{i j k l} \epsilon_{k l}$. where $\sigma$ is stress tensor and $\epsilon$ is strain tensor (both of rank 2).

There appears to be a relation between the hydrogen bonding and the elastic constant since [011] crystallographic direction (corresponding to $\mathrm{C}_{y y z z}$ ) shows relatively high value. There are other factors such as intermolecular interaction, crystal packing, etc., which also play a role in the value of $\mathrm{C}_{i j k l}$. But in crystals with hydrogen bonding, it appears that hydrogen bonding also has a role in the elastic tensor components. Note that the diagonal elements are the largest. Of these the element $\mathrm{C}_{y y y y}$ are the largest.

\section{Conclusion}

The present study investigates the properties of the triclinic phase of malonic acid. Of the two employed intermolecular potentials, MAL gave reasonable agreement of many of the static properties such as lattice constants. We have also computed the relaxation times of hydrogen bond chains along [011] direction in the triclinic phase. It is seen that longer the chain, shorter its relaxation time. Increase in temperature also leads to reduced relaxation time. The relaxation times vary between approximately $19 \mathrm{ps}$ for $l=5$ at $350 \mathrm{~K}$ to 566 ps for $l=1$ at $250 \mathrm{~K}$. These are likely to be important for proton conduction in the solid phase. In addition to these the elements of the second-rank elastic constant tensor have been computed from molecular dynamics simulations. There are in all 21 independent values for these. Within the error in the computed elastic constants, the elastic constant tensor is symmetric. The values of the elements are determined by the packing along different directions within the crystal as well as the hydrogen bonding.

The large elastic constant $\mathrm{C}_{y y z z}$ and the presence of hydrogen bond along [011] appears to support the findings by Azuri et al who suggested that there is a relation between the direction of the hydrogen bond chains and the elastic constant. Directions along which there are hydrogen bond chains appear to show higher elastic constants. We are presently investigating the role of interchain interactions and its implication for the elastic constant tensor.

\section{Acknowledgements}

S. S. R. R. Perumal and SY thanks financial support through TUE-CMS project of DST at IISc, and acknowledges the computational resources of Cray X40 at SERC-IISc, and TUE-CMS.

\section{References}

1. Azuri I, Meirzadeh E, Ehre D, Cohen S R, Rappe A M, Lahav M, Lubomirsky I and Kronik L 2015 Unusually large youngs moduli of amino acid molecular crystals Angew. Chem. Int. Ed. Engl. 5413566

2. Hu X, Zhou J, Vatankhah-Varnosfaderani M, Daniel W F M, Li Q, Zhushma A P, Dobrynin A V and Sheiko S S 2016 Programming temporal shapeshifting Nat. Commun. 712919

3. Monk J D, Bucholz E W, Boghozian T, Deshpande S, Schieber J, Bauschlicher Jr C W and Lawson J W 2015 Computational and experimental study of phenolic resins: Thermal-mechanical properties and the role of hydrogen bonding Macromolecules $\mathbf{4 8} 7670$

4. Davies E, Duer M J, Ashbrook S E and Griffin J M 2012 Applications of nmr crystallography to problems in biomineralization: refinement of the crystal structure and $31_{\mathrm{p}}$ solid-state $\mathrm{nmr}$ spectral assignment of octacalcium phosphate J. Am. Chem. Soc. 13412508

5. Dharmawardhana C, Bakare M, Misra A and Ching W Y 2016 Nature of interatomic bonding in controlling the mechanical properties of calcium silicate hydrates $J$. Am. Chem. Soc. 992120

6. Geiger A, Stillinger F H and Rahman A 1979 Aspects of the percolation process for hydrogen-bond networks in water J. Am. Chem. Soc. 704185

7. Geiger A, Mausbach P, Schnitker J, Blumberg R L and Stanley H E 1984 Structure and dynamics of the hydrogen bond network in water by computer simulations $J$. Phys. Colloq. $45 \mathrm{C} 7$

8. Kaur R, Perumal S S R R, Bhattacharyya A B, Yashonath S and Guru Row T N 2014 Structural insights into proton conduction in gallic acid-isoniazid cocrystals Cryst. Growth Des. 14423 
9. Stanley $\mathrm{H}$ E and Teixeira $J 1980$ Interpretation of the unusual behavior of $\mathrm{H}_{2} \mathrm{O}$ at low temperatures: tests of a percolation model J. Chem. Phys. 733404

10. Etter M C, MacDonald J C and Bernstein J 1990 Graphset analysis of hydrogen-bond patterns in organic crystals Acta Crystallogr., Sect. B: Struct. Sci. 46256

11. Bernstein J, Davis R E, Shimoni L and Chang N L 1995 Patterns in hydrogen bonding: functionality and graph set analysis in crystals Angew. Chem., Int. Ed. 341555

12. Medhekar N V, Ramasubramaniam A, Ruoff R S and Shenoy V B 2010 Hydrogen bond networks in graphene oxide composite paper: structure and mechanical properties Acs Nano 42300

13. Dikin D A, Stankovich S, Zimney E J, Piner R D, Dommett G H B, Evmenenko G, Nguyen S T and Ruoff R S 2007 Preparation and characterization of graphene oxide paper Nature $\mathbf{4 4 8} 457$

14. Ribeiro da Silva M A V, Monte M J S and Ribeiro J R 1999 Vapour pressures and the enthalpies and entropies of sublimation of five dicarboxylic acids J. Chem. Thermodyn. 311093

15. Petit L, Lapalu L and Sautet P 2009 Self-assembly of diacid molecules: A theoretical approach of molecular interactions J. Phys. Chem. C 11317566

16. Meier B H, Graf F and Ernst R R 1982 Structure and dynamics of intramolecular hydrogen bonds in carboxylic acid dimers: A solid state nmr study J. Chem. Phys. 76767

17. Teixeira-Dias J J C and Fausto R 1986 A molecular mechanics force field for conformational analysis of simple acyl chlorides, carboxylic acids and esters J. Molec. Struct. 144199

18. Thalladi V R, Nüsse M, and Boese R 2000 The melting point alternation in $\alpha$-alkanedicarboxylic acids $J$. Am. Chem. Soc. 1229227

19. Mishra M K, Varughese S, Ramamurty U and Desiraju G R 2013 Odd-even effect in the elastic modulii of $\alpha$ alkanedicarboxylic acids J. Am. Chem. Soc. 1358121

20. Desiraju G R 1996 The ch *.. o hydrogen bond: structural implications and supramolecular design Acc. Chem. Res. 29441

21. Steiner T 1996 C [sbnd] h [sbnd] o hydrogen bonding in crystals Crystallogr. Rev. 61

22. Yellin Z B and Leiserowitz L 1984 The role played by $\mathrm{c}-\mathrm{ho}$ and $\mathrm{c}-\mathrm{hn}$ interactions in determining molecular packing and conformation Acta Crystallogr., Sect. B: Struct. Sci. 40159

23. Taylor R and Kennard O 1982 Crystallographic evidence for the existence of ch. cntdot.. cntdot.. cntdot. o, ch. cntdot.. cntdot.. cntdot. $\mathrm{n}$ and ch. cntdot.. cntdot.. cntdot. cl hydrogen bonds J. Am. Chem. Soc. 1045063

24. Parrinello M and Rahman A 1980 Crystal structure and pair potentials: A molecular-dynamics study Phys. Rev. Lett. 451196
25. Hoover W G 1985 Canonical dynamics: equilibrium phase-space distributions Phys. Rev. A 311695

26. Ma X, Chakraborty P, Henz B J and Zachariah M R 2011 Molecular dynamic simulation of dicarboxylic acid coated aqueous aerosol: structure and processing of water vapor Phys. Chem. Chem. Phys. 139374

27. Todorov I T, Smith W, Trachenko K, and Dove M T 2006 Dl_poly_3: new dimensions in molecular dynamics simulations via massive parallelism J. Mater. Chem. 16 1911

28. Gao G, Van Workum K, Schall J D and Harrison J A 2006 Elastic constants of diamond from molecular dynamics simulations J. Phys.: Condens. Matter 18 S1737

29. Schall J D, Gao G and Harrison J A 2008 Elastic constants of silicon materials calculated as a function of temperature using a parametrization of the secondgeneration reactive empirical bond-order potential Phys. Rev. B 77115209

30. Goedkoop J A and MacGillavry C H 1957 The crystal structure of malonic acid Acta Crystallogr. 10125

31. Ganguly S, Fernandes J R, Desiraju G R and Rao CNR 1980 Phase transition in malonic acid: An infrared study Chem. Phys. Lett. 69227

32. Tong C, Blanco M, Goddard W A and Seinfeld J H 2004 Thermodynamic properties of multifunctional oxygenates in atmospheric aerosols from quantum mechanics and molecular dynamics: Dicarboxylic acids Environ. Sci. Technol. 383941

33. Jeffrey G A 1997 An introduction to hydrogen bonding (New York: Oxford university press)

34. Luzar A and Chandler D 1996 Hydrogen-bond kinetics in liquid water Nature 37955

35. Chandra A 2000 Effects of ion atmosphere on hydrogenbond dynamics in aqueous electrolyte solutions Phys. Rev. Lett. 85768

36. Matsumoto M, Saito S and Ohmine I 2002 Molecular dynamics simulation of the ice nucleation and growth process leading to water freezing Nature $\mathbf{4 1 6} 409$

37. Chandra A and Chowdhuri S 2001 Effects of hydrogenbond environment on single particle and pair dynamics in liquid water J. Chem. Sci. 113591

38. Pradhan T, Ghoshal P and Biswas R 2008 Structural transition in alcohol-water binary mixtures: A spectroscopic study J. Chem. Sci. 120275

39. Buldyrev S V, Kumar P, Debenedetti P G, Rossky P J, and Stanley H E 2007 Water-like solvation thermodynamics in a spherically symmetric solvent model with two characteristic lengths Proc. Natl. Acad. Sci. U. S. A. 10420177

40. Kumar P, Han S and Stanley H E 2009 Water-like solvation thermodynamics in a spherically symmetric solvent model with two characteristic lengths J. Phys.: Condens. Matter 21504108 